ARTICLE

\title{
Chaos-assisted two-octave-spanning microcombs
}

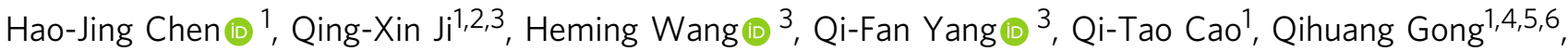 \\ Xu Yi (i) ${ }^{2,7 凶} \&$ Yun-Feng Xiao (1) ${ }^{1,4,5,6 凶}$
}

Since its invention, optical frequency comb has revolutionized a broad range of subjects from metrology to spectroscopy. The recent development of microresonator-based frequency combs (microcombs) provides a unique pathway to create frequency comb systems on a chip. Indeed, microcomb-based spectroscopy, ranging, optical synthesizer, telecommunications and astronomical calibrations have been reported recently. Critical to many of the integrated comb systems is the broad coverage of comb spectra. Here, microcombs of more than two-octave span ( $450 \mathrm{~nm}$ to $2,008 \mathrm{~nm}$ ) is demonstrated through $\chi^{(2)}$ and $\chi^{(3)}$ nonlinearities in a deformed silica microcavity. The deformation lifts the circular symmetry and creates chaotic tunneling channels that enable broadband collection of intracavity emission with a single waveguide. Our demonstration introduces a new degree of freedom, cavity deformation, to the microcomb studies, and our microcomb spectral range is useful for applications in optical clock, astronomical calibration and biological imaging.

\footnotetext{
${ }^{1}$ State Key Laboratory for Mesoscopic Physics and Frontiers Science Center for Nano-optoelectronics, School of Physics, Peking University, 100871 Beijing, China. ${ }^{2}$ Department of Electrical and Computer Engineering, University of Virginia, Charlottesville, VA 22904, USA. ${ }^{3}$ T. J. Watson Laboratory of Applied Physics, California Institute of Technology, Pasadena, CA 91125, USA. ${ }^{4}$ Collaborative Innovation Center of Quantum Matter, 100871 Beijing, China. ${ }^{5}$ Collaborative Innovation Center of Extreme Optics, Shanxi University, 030006 Taiyuan, China. ${ }^{6}$ Peking University Yangtze Delta Institute of Optoelectronics, Nantong, Jiangsu 226010, China. ${ }^{7}$ Department of Physics, University of Virginia, Charlottesville, VA 22904, USA. ${ }^{凶}$ email: yi@virginia.edu; yfxiao@pku.edu.cn
} 
$\mathrm{M}$ icroresonator-based frequency comb (microcomb) uses Kerr nonlinearity to create parametric gain and offset cavity loss ${ }^{1}$. It has been demonstrated in various material platforms including silica ${ }^{2}, \mathrm{CaF}_{2}$ (ref. $\left.{ }^{3}\right), \mathrm{MgF}_{2}$ (ref. ${ }^{4}$ ), high-index silica ${ }^{5}$, silicon nitride ${ }^{6}, \mathrm{AlN}^{7}$, diamond ${ }^{8}$, and $\mathrm{LiNbO}_{3}{ }^{9}$. The recent development of dissipated Kerr soliton microcombs ${ }^{10-17}$ has enabled applications ranging from spectroscopy to astronomy calibration ${ }^{18-25}$. The microcomb also provides a novel platform for nonlinear physics studies and has led to observations of Stokes solitons ${ }^{26}$, soliton crystals $^{27}$, and soliton interactions ${ }^{28}$. In these demonstrations, the microcombs are generated in either circular-symmetric whispering-gallery-mode (WGM) cavities or waveguide-mode cavities, and are coupled out by phase-matched, evanescent couplers. The efficiency of the coupler drops significantly at wavelengths far from that of the pump laser. The same challenge is also imposed on microcavity harmonic generation, but it is often overcome by adding additional coupler ${ }^{29}$. However, this resolution does not directly applied to microcombs with broad spectral coverage. Complicated coupler has recently been designed and tested for octavespanning microcombs ${ }^{30,31}$.

Asymmetric (deformed) cavity, first proposed by Nöckel and Stone ${ }^{32}$, can output emissions from whispering-gallery modes to free space without using evanescent couplers. Its applications in directional laser ${ }^{33}$, suppression of laser spatiotemporal instabilities $^{34}$, light storage ${ }^{35}$, as well as single nanoparticle biosensing ${ }^{36}$ have been demonstrated. Generally, ray dynamics in a billiard together with the dynamic tunneling in the momentum space provides a versatile tool to understand the interesting phenomena in asymmetric microcavities ${ }^{37,38}$. Recently, it was revealed that the chaotic channels in the deformed cavity can assist broadband momentum transformation ${ }^{39}$, opening up new possibilities to resolve the challenge in outputting broadband nonlinear emissions from microcavities.

In this Article, we report a microcomb spanning from 450 to $2008 \mathrm{~nm}$ in a deformed cavity (Fig. 1a). The microcomb is pumped near $1550 \mathrm{~nm}$, and its frequency range is extended by nonlinearities ${ }^{40-44}$ of intracavity $\chi^{(3)}$ and symmetry-breaking induced $\chi^{(2)}$ (ref. ${ }^{45}$ ) (Fig. 1c). Importantly, to output the broadband emission, the microcavity is deformed slightly to create a chaotic tunneling channel. Because of the chaotic motion, the angular momentum of light is not conserved, and it changes with time, covering a broad range of values (Fig. 1b). Once the angular momenta of the comb reach the corresponding critical lines of total internal reflections, the comb can be effectively collected by a nanofiber through nearly wavelength-independent refraction process ${ }^{39}$.

\section{Results}

Two-octave-spanning microcomb. The silica microtoroid resonator ${ }^{46}$ used in this experiment has principal (minor) diameter of $75 \mu \mathrm{m}(4.2 \mu \mathrm{m})$ and its $Q$-factor is $1.6 \times 10^{7}$ at $1550 \mathrm{~nm}$. The microcavity is weakly deformed to create chaotic channels and the minimum diameter is $5.6 \%$ smaller than the maximum diameter. The details of the deformed cavity boundary shape are included in the "Methods" section. A tunable laser at $1550 \mathrm{~nm}$ is used as the pump. Its power is amplified to $1.6 \mathrm{~W}$ and is coupled into the cavity through a 720 -nm-diameter nanofiber.

The optical spectrum of the microcomb is presented in Fig. 2a. The frequency span of the microcomb ranges from 150 to 670 THz. An optical spectrum analyzer (OSA, Yokogawa) is used to measure the spectrum above $1200 \mathrm{~nm}$ (shown in red), while the shorter wavelength part (shown in blue) is measured by a spectrograph (Andor). The power of the OSAs are calibrated at 1550 and $635 \mathrm{~nm}$. The selection of the calibration wavelength is
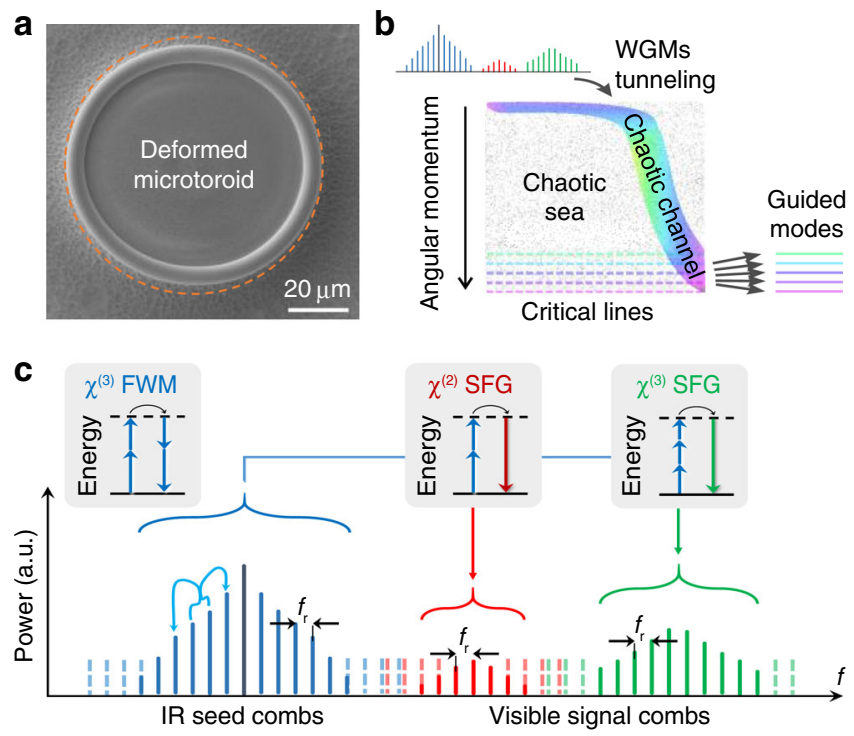

Fig. 1 Schematic for broadband microcombs in the deformed microcavity. a Scanning electron microscope image of a deformed microresonator. As a comparison, a circular contour is shown in dashed orange line. $\mathbf{b}$ Conceptual illustration of chaos-assisted momentum transformation and dynamical tunneling from whispering-gallery-modes (WGMs) to chaotic states. c Schematic of the nonlinear frequency conversion from infrared (IR) to visible wavelengths in the deformed cavity with four-wave mixing (FWM) and sum-frequency generation (SFG, including harmonic generations as a special case).

constrained by the collection of laser sources in our lab. The power mismatch at $1200 \mathrm{~nm}$ is likely to come from the variation of OSA's efficiency versus wavelength. The noise floor of the spectrograph is also well characterized. The violet and green emission from the microtoroid can be observed directly by a CCD camera set on the side of the microtoroid (Fig. 2a, inset). The zoom-in spectra of the microcombs at 378.3-389.4, 480-491.1, 549.3-560.4, and 647.4-658.5 THz are shown in Fig. 2b-e.

The formation process of broadband microcomb in deformed cavity is investigated both experimentally and numerically (Fig. 3). In this measurement, the frequency detuning of laser cavity is slowly decreasing while the optical spectrum is recorded. Four typical phases of microcomb are presented in Fig. 3. First, the thresholdless harmonic generation is observed at 386.9 and 580.5 $\mathrm{THz}$ as the frequency of pump light is $193.4 \mathrm{THz}$. With the decrease of detuning, the intracavity pump power reaches the parametric threshold and the primary comb lines are generated near the pump wavelength as well as the harmonic wavelengths. We refer the comb lines near the pump wavelength as seed comb, and the rest of the comb as signal comb. The signal comb is generated from sum-frequency generation (SFG) of the seed comb, and the four-wave mixing between the seed comb and the harmonics of the pump laser. Subcombs with comb spacing identical to cavity FSR appear in both seed comb and signal comb when further decreasing the detuning. The subcombs will eventually merge together in all three bands. The formation process of broadband microcomb is well reproduced in our numerical simulation of coupled Lugiato-Lefever (LL) equations (see Methods) and is presented in Fig. 3b. Note that the numerical simulation of intracavity light field is performed by employing a circular cavity without the effects of chaotic propagation. This configuration is an approximation for the weakly deformed high$Q$ cavity to reduce computational complexity.

A comparison of comb output between phase-matched coupling and chaos-assisted coupling is demonstrated. In this 
a Wavelength $(\mathrm{nm})$

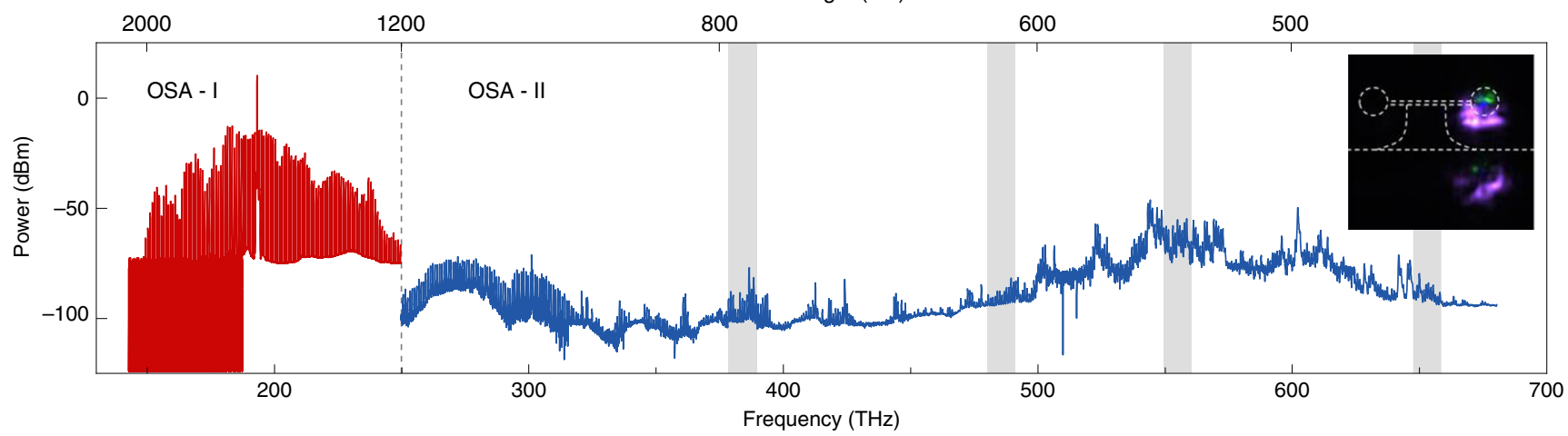

b Wavelength $(\mathrm{nm})$

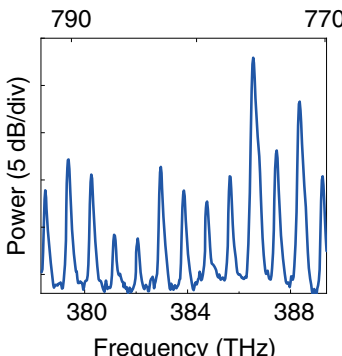

C Wavelength $(\mathrm{nm})$

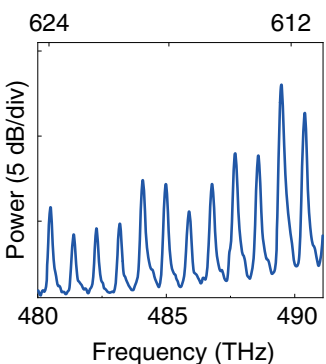

d Wavelength $(\mathrm{nm})$

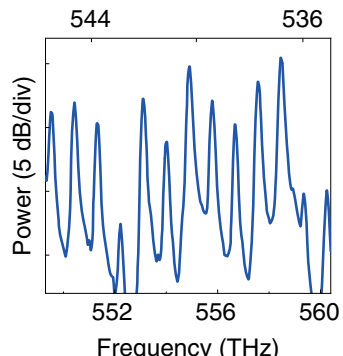

e Wavelength $(\mathrm{nm})$

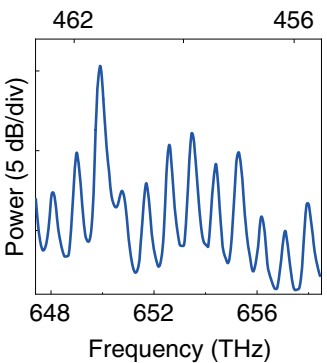

Fig. 2 Optical spectrum of two-octave-spanning microcombs. a Complete optical spectrum of microcombs. Spectrum measured with Yokogawa optical spectrum analyzer (OSA) above $1200 \mathrm{~nm}$ is presented in red, while the shorter wavelength part is measured by an Andor spectrograph (shown in blue). The absolute power of the spectrum are calibrated at 1550 and $635 \mathrm{~nm}$ for the OSA and spectrograph, respectively. The power mismatch of the red and blue spectrum at $1200 \mathrm{~nm}$ is caused by the spectrograph efficiency variation over the broad spectral range. Inset: an image taken by a CCD camera on the side of the cavity. Green and violet emissions are apparent. b-e Zoom-in spectra of the gray areas are shown in panel $\mathbf{a}$. The frequency ranges for $\mathbf{b}$-e are 378.3-389.4, 480-491.1, 549.3-560.4, and 647.4-658.5 THz.

a

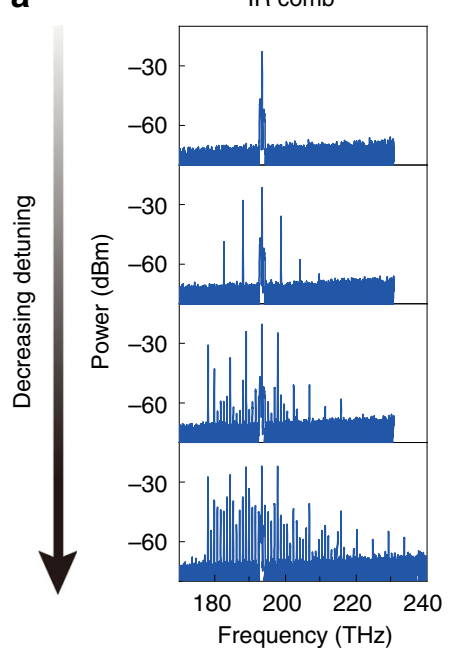

$\chi^{(2)} \mathrm{comb} \quad \chi^{(3)} \mathrm{comb}$

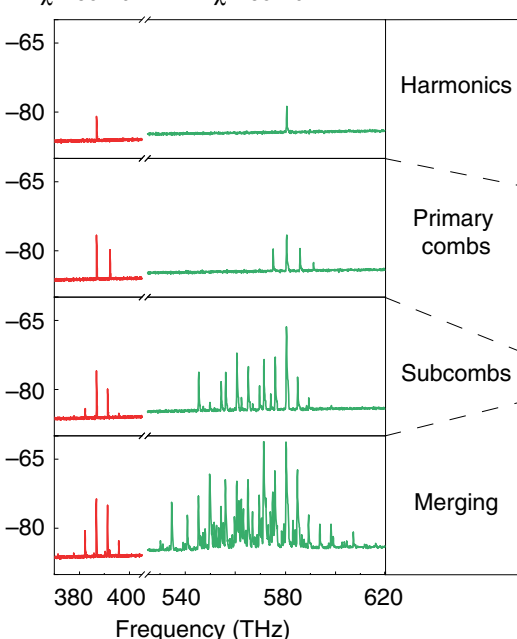

b IR comb

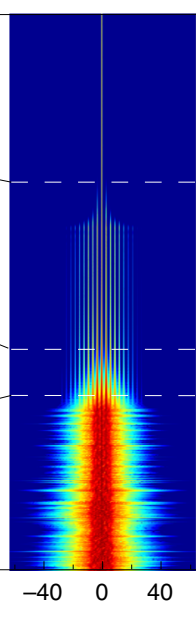

$\chi^{(2)} \operatorname{comb}$

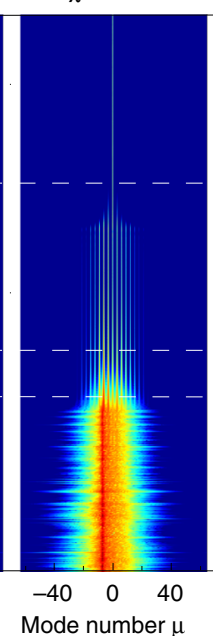

$\chi^{(3)}$ comb

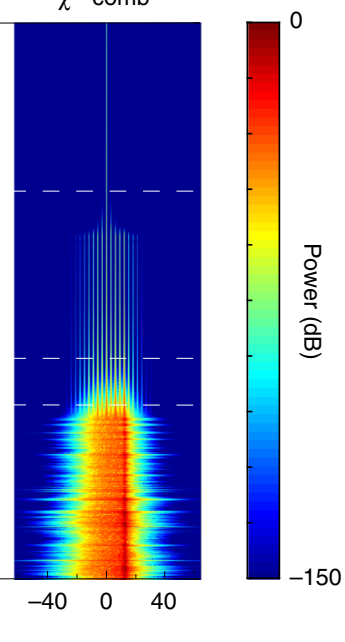

Fig. 3 Formation process of microcombs. a Four representative optical spectra of microcombs. The amplitude of cavity-laser detuning is gradually decreased from top to bottom. The second and third harmonic generation is observed first, followed by the primary combs in all three frequency bands. Subcombs are then generated and they slowly merge with the decrease of detuning. $\mathbf{b}$ Simulated microcomb formation process. Pump detuning is scanned from shorter to longer wavelength, and the spectrum evolution of microcomb is presented. The intracavity power for three bands is normalized to the largest value of their own bands.

experiment, a microfiber and a nanofiber are simultaneously coupled to the deformed cavity (Fig. 4a). The diameter of the microfiber is set to $1.5 \mu \mathrm{m}$ to achieve phase-matched coupling with the whispering-gallery mode at $1550 \mathrm{~nm}$. The $0.5-\mu \mathrm{m}-$ diameter nanofiber has effective refractive index close to 1 , and it is used to collect the comb light through chaos-assisted coupling. The coupling efficiency can be simulated by 3D FDTD method, and the result is presented in Fig. $4 \mathrm{~b}$. The efficiency for phasematched coupling decreases rapidly with the increase of optical frequency, while the efficiency for chaos-assisted coupling 
a

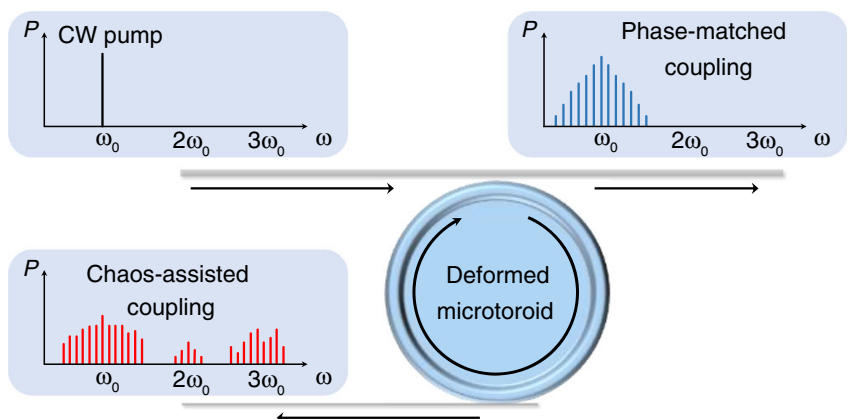

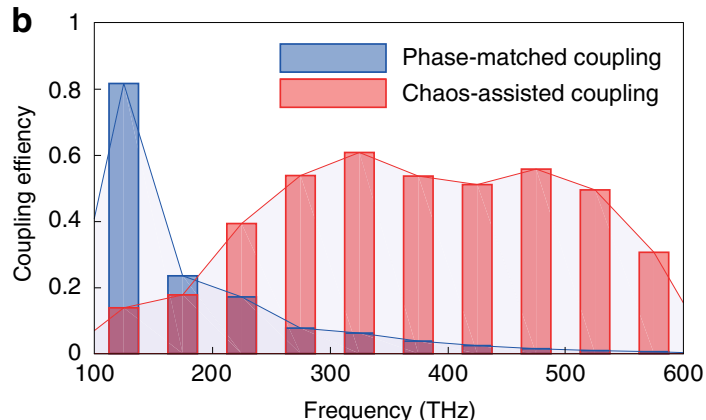
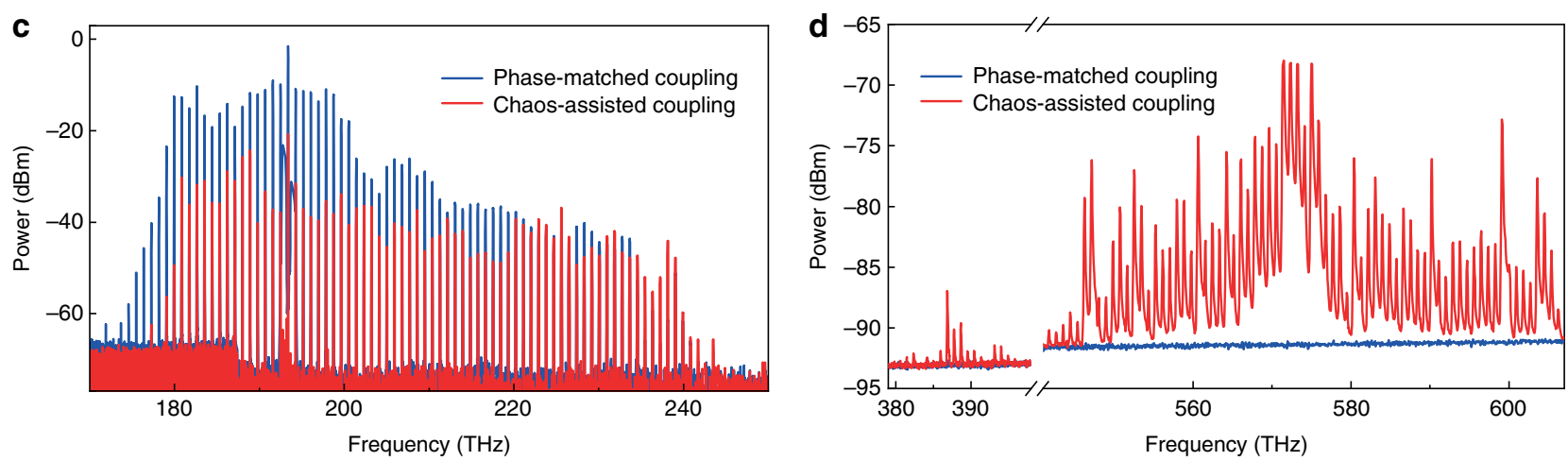

Fig. 4 Comparison between chaos-assisted coupling combs and phase-matched coupling combs. a Conceptual schematic: a phase-matched microfiber is used to couple pump laser into the deformed cavity and it also collects comb emissions at the through port. A nanofiber is used at the drop port to output comb light through chaos-assisted coupling. $\mathbf{b}$ Three-dimensional finite difference time-domain (3D FDTD) simulation of the coupling efficiencies of both phase-matched coupling and chaos-assisted coupling. c, $\mathbf{d}$ Optical spectra of the frequency combs with phase-matched coupling (blue) and chaos-assisted coupling (red) in infrared (c) and visible (d) regions.

maintains very well from near-IR to visible wavelengths. The dimension of the cavity is scaled down in this simulation and the detailed is described in the Methods. In the experiment, $300 \mathrm{~mW}$ pump power is sent into the cavity through the microfiber. The optical spectra are measured at both the through port and drop port and are shown in Fig. 4c, d. No comb signal in near-visible or visible band can be seen for the phase-matched coupling output. In comparison, intense comb lines are detected with chaos-assisted coupling.

Observation of soliton formation steps. Soliton formation in microresonators is critical to many microcomb applications for its high coherence and predictable spectrum envelope ${ }^{16}$. Here, we show that soliton formation could be feasible in a deformed cavity for the first time. In the experiment, the laser wavelength is scanned from blue-detuned to red-detuned regime, while the comb power is recorded on the oscilloscope. When the laser scanning rate is increased to $\sim 10 \mathrm{~nm} / \mathrm{s}$, the distinct soliton formation steps are observed. Upon entering the soliton regime, the comb power shows a "step" feature and the noise on the comb power disappears. Both single and multiple steps are observed and presented in Fig. 5. This points to evidence of dissipated Kerr cavity solitons in a microresonator ${ }^{10}$. It shall be noted that the transition from noisy comb to soliton regime is often complicated by thermal effect. The abrupt change in comb power leads to a rapid decrease of cavity temperature and thus a blueshift of the resonator frequency. As a result, the cavity-laser detuning quickly exits the soliton existence range and thus the soliton steps in our experiment are only $\sim 1 \mu$ s in duration. Several methods exist to overcome this thermal complexity, including pump power kicking 47 , rapid frequency scanning by using single-sideband
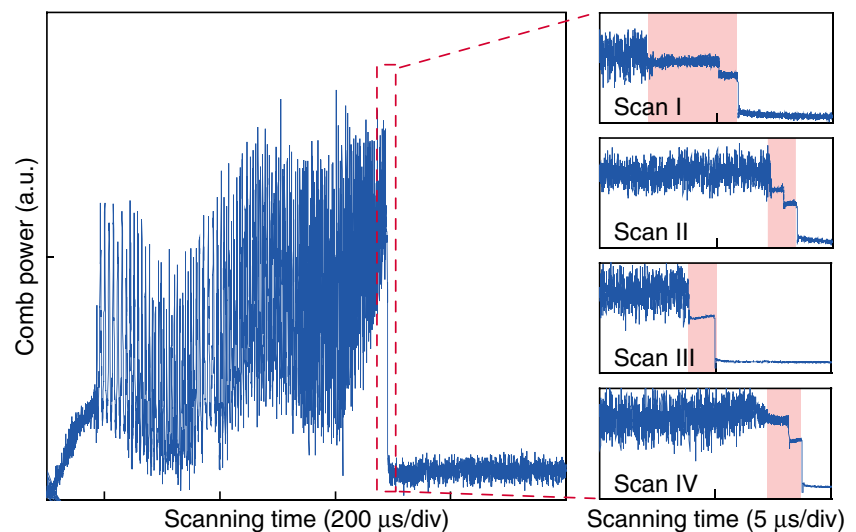

Fig. 5 Observation of soliton formation steps. Experimental traces of the comb power are recorded when the pump laser frequency is scanned from blue to red detuning at a scanning speed of $\sim 10 \mathrm{~nm} / \mathrm{s}$. The right panels show the zoom-in traces of four representative soliton formation steps in different scans, of which the number and the amplitude of steps change from scan to scan.

modulator $^{48}$, and auxiliary laser for thermal compensation ${ }^{49,50}$. Further investigation using these methods is needed in the future to stably access the soliton state in our cavity and to verify soliton mode-locking.

\section{Discussion}

In summary, we have demonstrated microcomb with two-octave span ranging from 450 to $2008 \mathrm{~nm}$ in a deformed cavity. The microcomb wavelength range is useful for optical clock, medical 
imaging, and calibrating radial velocity for exoplanet detection. The deformation of cavity lifts the circular symmetry and introduces a new degree of freedom to the microcomb systems. This could be used as an additional knob for broadband dispersion engineering of microresonator. Preliminary indication of soliton formation is also shown in the deformed cavity. The combination of temporal solitons and chaos in deformed cavity in the future may provide new possibilities for optical soliton physics studies and microcomb applications.

\section{Methods}

Deformed cavity design. In a deformed cavity, the angle of reflection and the angle of following incidence are different when a light beam is reflected on the boundary of the cavity. As a result, the incident angle $\chi$ of reflection is not conserved, nor is the angular momentum quantity $\sin \chi$. This varying $\chi$ makes the intracavity ray dynamics highly sensitive to initial conditions, and the chaotic channel forms accompanied by rapid and broadband momentum transformation.

By using this momentum transformation, the effective mode index of light can be reduced from 1.44 (close to that of the WGMs) to nearly 1 (close to that of the nanofiber) in the chaotic channel. As a result, the light can be easily coupled into the nanofiber from the chaotic channel through the wavelength-independent refraction. This chaos-assisted broadband momentum transformation is provided in the cavity with its boundary designed as

$$
R(\phi)=\left\{\begin{array}{c}
R_{0}+R_{0} \sum_{i=2,3} a_{i} \cos ^{i} \phi \cos \phi \geq 0 \\
R_{0}+R_{0} \sum_{i=2,3} b_{i} \cos ^{i} \phi \cos \phi<0
\end{array},\right.
$$

where $R_{0}$ is the size parameter, and $a(b)_{2,3}$ are optimized as $a_{2}=-0.1329, a_{3}=$ $0.0948, b_{2}=-0.0642$, and $b_{3}=-0.0224$.

\section{Coupled LL equations in the presence of $\chi^{(2)}$ and $\chi^{(3)}$ nonlinear processes. The} comb formation process is simulated with three normalized coupled LL equations, which include multiple $\chi^{(2)}$ and $\chi^{(3)}$ nonlinear effects, e.g. SFG, different frequency generation, self-phase modulation, and cross-phase modulation. In the equations, $\mu_{a b}$ and $\mu_{a c}$ describe coupling strength for $\chi^{(2)}$ and $\chi^{(3)} \mathrm{SFG} ; \mu_{b a}$ and $\mu_{c a}$ for the reverse process; $\zeta_{a b}$ and $\zeta_{a c}$ denote cross-phase modulation for $\chi^{(2)}$ and $\chi^{(3)}$ combs, and the inverse process is described by $\zeta_{b a}$ and $\zeta_{c a}$. Self-phase modulation of $\chi^{(2)}$ and $\chi^{(3)}$ combs is described by $\zeta_{b}$ and $\zeta_{c}$. The coupled LLEs are as follows:

$\frac{\partial}{\partial \tau} \Phi_{a}=\left(-1-i \alpha_{a}+i \beta_{a} \frac{\partial^{2}}{\partial \phi^{2}}+i\left|\Phi_{a}\right|^{2}+i \zeta_{b a}\left|\Phi_{b}\right|^{2}+i \zeta_{c a}\left|\Phi_{c}\right|^{2}\right) \Phi_{a}+f_{0}+i \mu_{b a} \Phi_{a}^{*} \Phi_{b}+i \mu_{c a} \Phi_{a}^{* 2} \Phi_{c}$,

$$
\frac{\partial}{\partial \tau} \Phi_{b}=\left(-\frac{\kappa_{b}}{\kappa_{a}}-i \alpha_{b}-\Delta k_{b} \frac{\partial}{\partial \phi}+i \beta_{b} \frac{\partial^{2}}{\partial \phi^{2}}+i \zeta_{b}\left|\Phi_{b}\right|^{2}+i \zeta_{a b}\left|\Phi_{a}\right|^{2}\right) \Phi_{b}+i \mu_{a b} \Phi_{a}^{2},
$$

$$
\frac{\partial}{\partial \tau} \Phi_{c}=\left(-\frac{\kappa_{c}}{\kappa_{a}}-i \alpha_{c}-\Delta k_{c} \frac{\partial}{\partial \phi}+i \beta_{c} \frac{\partial^{2}}{\partial \phi^{2}}+i \zeta_{c}\left|\Phi_{c}\right|^{2}+i \zeta_{a c}\left|\Phi_{a}\right|^{2}\right) \Phi_{c}+i \mu_{a c} \Phi_{a}^{3},
$$

where the electromagnetic field in the cavity is described by three slowly-varying field envelopes $\Phi_{a, b, c}$ for (a) infrared, (b) $\chi^{(2)}$, and (c) $\chi^{(3)}$ combs, respectively. The envelope $\Phi_{a, b, c}$ is normalized to $\left|\Phi_{a, b, c}\right|^{2}=2 g_{a, b, c} N_{a, b, c} / \kappa_{a, b, c}$, where $N_{a, b, c}$ is photon number in the corresponding fields. $\kappa_{a, b, c}$ is the decay rate of the comb modes and $g_{a, b, c}=n_{2} c \hbar \omega_{a, b, c}^{2} /\left(n_{a, b, c}^{2} V_{a, b, c}\right)$, where $\hbar$ is the Planck's constant, $c$ is the speed of light in vacuum, $n_{2}$ is the Kerr nonlinear index, $\omega_{a, b, c}$ is the resonance frequency at the center of each frequency band, $n_{a, b, c}$ is the refractive index, and $V_{a, b, c}$ is the effective mode volume. The envelopes are functions of $\tau$ and $\phi$, where $\tau$ is the normalized time, $\tau=\kappa_{\mathrm{a}} t / 2, t$ is the lab time, and $\phi$ is the cavity polar angle. $\alpha_{a, b, c}$ denotes normalized detuning and $f_{0}$ is the normalized pump input. $\beta_{a, b, c}$ denotes normalized second-order chromatic dispersion, and $\Delta k_{b c}$ is the FSR mismatch between infrared combs and $\chi^{(2)}\left(\chi^{(3)}\right)$ combs in the unit of half cavity linewidth.

Parameters used in the LLE simulation are: $\kappa_{a} / 2 \pi=1.21 \times 10^{7} \mathrm{~Hz}, \kappa_{b} / 2 \pi=$ $2.03 \times 10^{7} \mathrm{~Hz}, \kappa_{c} / 2 \pi=3.05 \times 10^{7} \mathrm{~Hz}, \beta_{a}=1.8565, \beta_{b}=-4.1258, \beta_{c}=-6.0227$, $\Delta k_{b}=-1.0179 \times 10^{3}, \Delta k_{c}=-3.2310 \times 10^{3}, \zeta_{b}=1.6840, \zeta_{c}=2.5274, \zeta_{b a}=1.8738$, $\zeta_{c a}=1.1729, \zeta_{a b}=9.2780, \zeta_{a c}=13.0269, \mu_{b a}=0.0058, \mu_{c a}=0.2139, \mu_{a b}=0.0143$, $\mu_{a c}=0.7921, f_{0}=26.1562$. Parameters derived from experimental measurements are $\kappa_{a, b, c}$, while other parameters are derived from 2D finite element method (FEM) simulation with rotation symmetry.

3D FDTD simulations of broadband coupling efficiency. The full 3D simulations of coupling efficiency are performed by a commercial simulator based on FDTD method. The microtoroid resonator is placed on the $x-y$ plane. To make the simulation time affordable, the principal and minor diameters of the microresonator are scaled down to 24 and $4 \mu \mathrm{m}$, respectively. The thickness of the central disk is $2 \mu \mathrm{m}$. The nanofiber and the microfiber are represented by cylinders with diameters of 0.5 and $1.5 \mu \mathrm{m}$. A probe light source is applied to one side of the fiber.
The excited mode in the fiber is set to be a fundamental mode. The material of the resonator is set to be silica with an additional imaginary part of $10^{-5}$ to its refractive index, and the material of the fiber is set to be silica without extra loss. Coupling efficiency is calculated as the difference of the base value and the dip value of the transmission spectrum. Different resonant modes are identified by field monitors inside the cavity. In Fig. 4b, a fundamental TE mode is used to calculate the coupling efficiency.

\section{Data availability}

Source data for Fig. 2 to Fig. 5 can be accessed at https://doi.org/10.6084/m9. figshare.12030408. Additional information is available from the corresponding authors upon reasonable request.

\section{Code availability}

The codes that support the findings of this study are available from the corresponding authors upon reasonable request.

Received: 5 March 2019; Accepted: 3 April 2020; Published online: 11 May 2020

\section{References}

1. Kippenberg, T. J., Holzwarth, R. \& Diddams, S. Microresonator-based optical frequency combs. Science 332, 555-559 (2011).

2. Del'Haye, P. et al. Optical frequency comb generation from a monolithic microresonator. Nature 450, 1214-1217 (2007).

3. Savchenkov, A. A. et al. Tunable optical frequency comb with a crystalline whispering gallery mode resonator. Phys. Rev. Lett. 101, 093902 (2008).

4. Liang, W. et al. Generation of near-infrared frequency combs from a $\mathrm{MgF}_{2}$ whispering gallery mode resonator. Opt. Lett. 36, 2290-2292 (2011).

5. Razzari, L. et al. CMOS-compatible integrated optical hyper-parametric oscillator. Nat. Photonics 4, 41-45 (2010).

6. Levy, J. S. et al. CMOS-compatible multiple-wavelength oscillator for on-chip optical interconnects. Nat. Photonics 4, 37-40 (2010).

7. Jung, H., Xiong, C., Fong, K. Y., Zhang, X. \& Tang, H. X. Optical frequency comb generation from aluminum nitride microring resonator. Opt. Lett. 38, 2810-2813 (2013).

8. Hausmann, B., Bulu, I., Venkataraman, V., Deotare, P. \& Lončar, M. Diamond nonlinear photonics. Nat. Photonics 8, 369-374 (2014).

9. Wang, C. et al. Monolithic lithium niobate photonic circuits for Kerr frequency comb generation and modulation. Nat. Commun. 10, 1-6 (2019).

10. Herr, T. et al. Temporal solitons in optical microresonators. Nat. Photonics. 8 145-152 (2014)

11. Yi, X., Yang, Q.-F., Yang, K. Y., Suh, M.-G. \& Vahala, K. Soliton frequency comb at microwave rates in a high-Q silica microresonator. Optica 2, 1078-1085 (2015).

12. Brasch, V. et al. Photonic chip-based optical frequency comb using soliton Cherenkov radiation. Science 351, 357-360 (2016).

13. Wang, P.-H. et al. Intracavity characterization of micro-comb generation in the single-soliton regime. Opt. Express 24, 10890-10897 (2016).

14. Joshi, C. et al. Thermally controlled comb generation and soliton modelocking in microresonators. Opt. Lett. 41, 2565-2568 (2016).

15. Gong, Z. et al. High-fidelity cavity soliton generation in crystalline AlN microring resonators. Opt. Lett. 43, 4366-4369 (2018).

16. Kippenberg, T. J., Gaeta, A. L., Lipson, M. \& Gorodetsky, M. L. Dissipative Kerr solitons in optical microresonators. Science 361, eaan8083 (2018).

17. He, Y. et al. Self-starting bi-chromatic $\mathrm{LiNbO}_{3}$ soliton microcomb. Optica 6 1138-1144 (2019)

18. Suh, M.-G., Yang, Q.-F., Yang, K. Y., Yi, X. \& Vahala, K. J. Microresonator soliton dual-comb spectroscopy. Science 354, 600-603 (2016).

19. Marin-Palomo, P. et al. Microresonator-based solitons for massively parallel coherent optical communications. Nature 546, 274-279 (2017).

20. Suh, M.-G. \& Vahala, K. J. Soliton microcomb range measurement. Science 359, 884-887 (2018).

21. Trocha, P. et al. Ultrafast optical ranging using microresonator soliton frequency combs. Science 359, 887-891 (2018).

22. Spencer, D. T. et al. An optical-frequency synthesizer using integrated photonics. Nature 557, 81-85 (2018).

23. Yang, Q.-F. et al. Vernier spectrometer using counterpropagating soliton microcombs. Science 363, 965-968 (2019).

24. Suh, M.-G. et al. Searching for exoplanets using a microresonator astrocomb. Nat. Photonics 13, 25-30 (2019)

25. Obrzud, E. et al. A microphotonic astrocomb. Nat. Photonics 13, 31 (2019).

26. Yang, Q.-F., Yi, X., Yang, K. Y. \& Vahala, K. Stokes solitons in optical microcavities. Nat. Phys. 13, 53-57 (2017). 
27. Cole, D. C., Lamb, E. S., Del'Haye, P., Diddams, S. A. \& Papp, S. B. Soliton crystals in Kerr resonators. Nat. Photonics 11, 671 (2017).

28. Yi, X., Yang, Q.-F., Yang, K. Y. \& Vahala, K. Imaging soliton dynamics in optical microcavities. Nat. Commun. 9, 3565 (2018).

29. Guo, X., Zou, C.-L. \& Tang, H. X. Second-harmonic generation in aluminum nitride microrings with $2500 \% / \mathrm{W}$ conversion efficiency. Optica 3, 1126-1131 (2016).

30. Li, Q. et al. Stably accessing octave-spanning microresonator frequency combs in the soliton regime. Optica 4, 193-203 (2017).

31. Moille, G. et al. Broadband resonator-waveguide coupling for efficient extraction of octave-spanning microcombs. Opt. Lett. 44, 4737-4740 (2019).

32. Nöckel, J. U. \& Stone, A. D. Ray and wave chaos in asymmetric resonant optical cavities. Nature 385, 45 (1997).

33. Shinohara, S. et al. Chaos-assisted directional light emission from microcavity lasers. Phys. Rev. Lett. 104, 163902 (2010).

34. Bittner, S. et al. Suppressing spatiotemporal lasing instabilities with wavechaotic microcavities. Science 361, 1225-1231 (2018).

35. Liu, C. et al. Enhanced energy storage in chaotic optical resonators. Nat. Photonics 7, 473 (2013).

36. Shao, L. et al. Detection of single nanoparticles and lentiviruses using microcavity resonance broadening. Adv. Mater. 25, 5616-5620 (2013).

37. Cao, H. \& Wiersig, J. Dielectric microcavities: model systems for wave chaos and non-hermitian physics. Rev. Mod. Phys. 87, 61 (2015).

38. Yang, J. et al. Pump-induced dynamical tunneling in a deformed microcavity laser. Phys. Rev. Lett. 104, 243601 (2010).

39. Jiang, X. et al. Chaos-assisted broadband momentum transformation in optical microresonators. Science 358, 344-347 (2017).

40. Jung, H., Stoll, R., Guo, X., Fischer, D. \& Tang, H. X. Green, red, and IR frequency comb line generation from single IR pump in AlN microring resonator. Optica 1, 396-399 (2014).

41. Miller, S. et al. On-chip frequency comb generation at visible wavelengths via simultaneous second- and third-order optical nonlinearities. Opt. Express 22, 26517-26525 (2014).

42. Wang, L. et al. Frequency comb generation in the green using silicon nitride microresonators. Laser Photon. Rev. 10, 631-638 (2016).

43. Xue, X. et al. Second-harmonic-assisted four-wave mixing in chipbased microresonator frequency comb generation. Light Sci. Appl. 6, e16253 (2017).

44. Fujii, S., Kato, T., Suzuki, R. \& Tanabe, T. Third-harmonic blue light generation from Kerr clustered combs and dispersive waves. Opt. Lett. 42, 2010-2013 (2017).

45. Zhang, X. et al. Symmetry-breaking-induced nonlinear optics at a microcavity surface. Nat. Photonics 13, 21 (2019).

46. Armani, D., Kippenberg, T., Spillane, S. \& Vahala, K. Ultra-high- $Q$ toroid microcavity on a chip. Nature 421, 925 (2003)

47. Yi, X., Yang, Q.-F., Youl, K. \& Vahala, K. Active capture and stabilization of temporal solitons in microresonators. Opt. Lett. 41, 2037-2040 (2016).

48. Stone, J. R. et al. Thermal and nonlinear dissipative-soliton dynamics in Kerr-microresonator frequency combs. Phys. Rev. Lett. 121, 063902 (2018).

49. Zhang, S. et al. Sub-milliwatt-level microresonator solitons with extended access range using an auxiliary laser. Optica 6, 206-212 (2019).
50. Zhou, H. et al. Soliton bursts and deterministic dissipative Kerr soliton generation in auxiliary-assisted microcavities. Light Sci. Appl. 8, 50 (2019).

\section{Acknowledgements}

The authors thank K. Vahala, B. Shen, L. Wu, Y. Zhi, P. Del'Haye, X.-C. Yu, L. Wang, Y.J. Qian, L. Shao, C.-H. Dong, J.-h. Chen, X.-F. Jiang, S.-X. Zhang, S.-J. Tang, L. Yao and L.-K. Chen for helpful discussions. This project is supported by the National Key R\&D Program of China (Grant No. 2016YFA0301302 and No. 2018YFB2200401), the National Natural Science Foundation of China (Grant Nos. 11825402, 11654003, 61435001, and 12041602), Beijing Academy of Quantum Information Sciences (Y18G20), Key R\&D Program of Guangdong Province (2018B030329001) and the Highperformance Computing Platform of Peking University. X.Y. is supported by U.S. National Science Fundation (award no. 1842641).

\section{Author contributions}

Y.-F.X. and X.Y. conceived the idea and designed the experiments. H.-J.C. fabricated the devices and built the experimental setup. H.-J.C., Q.-X.J., H.W., and Q.-F.Y. performed the measurements. Q.-X.J. and Q.-T.C. built the theoretical model and performed the simulations. All authors analyzed the data, participated in preparing the manuscript, and contributed to the discussions. Y.-F.X., X.Y., and Q.G. supervised the project.

\section{Competing interests}

The authors declare no competing interests.

\section{Additional information}

Correspondence and requests for materials should be addressed to X.Y. or Y.-F.X.

Peer review information: Nature Communications thanks the anonymous reviewers for their contribution to the peer review of this work.

Reprints and permission information is available at http://www.nature.com/reprints

Publisher's note Springer Nature remains neutral with regard to jurisdictional claims in published maps and institutional affiliations.

Open Access This article is licensed under a Creative Commons Attribution 4.0 International License, which permits use, sharing, adaptation, distribution and reproduction in any medium or format, as long as you give appropriate credit to the original author(s) and the source, provide a link to the Creative Commons license, and indicate if changes were made. The images or other third party material in this article are included in the article's Creative Commons license, unless indicated otherwise in a credit line to the material. If material is not included in the article's Creative Commons license and your intended use is not permitted by statutory regulation or exceeds the permitted use, you will need to obtain permission directly from the copyright holder. To view a copy of this license, visit http://creativecommons.org/ licenses/by/4.0/.

(C) The Author(s) 2020 